\title{
On contractibility of the Gelfand spectrum of semigroup measure algebras
}

\author{
A. R. Mirotin \\ amirotin@yandex.ru
}

\begin{abstract}
Sufficient conditions for a semigroup measure algebra to have contractible Gelfand spectrum are given and it is shown that for a wide class of semigroups these conditions are also necessary.
\end{abstract}

Key words: semitopological semigroup, measure algebra, Gelfand spectrum, contractibility, Hermite ring.

In the paper [1] by A. Sasane it was shown that the Gelfand spectrum (equipped with the Gelfand topology) of the algebra of complex bounded measures on the additive semigroup $[0,+\infty)$ is contractible. Several applications of this result are mentioned in [1], too. The main goal of this note is to give a simple proof of a generalization of the main result of [1] to general semigroups. Actually, we give sufficient conditions for a semigroup measure algebra $\mathcal{M}(S)$ to have contractible Gelfand spectrum $\Delta \mathcal{M}(S)$ and show that for a wide class of semigroups these conditions are also necessary. On the other hand, we show that for a nontrivial locally compact Abelian group $G$ the space $\Delta \mathcal{M}(G)$ is noncontractible.

In the following $S$ stands for an Abelian semitopological locally compact semigroup with unit $e$, and let $\mathcal{M}(S)$ be the algebra of all complex bounded regular Borel measures on $S$ with respect to the convolution (see, e.g. [2, Chapter 5])

$$
\int_{S} f d \mu * \nu:=\int_{S \times S} f(x y) d \mu(x) d \nu(y)\left(f \in C^{b}(S)\right) .
$$

Let $S_{1}^{*}$ denotes the semigroup of nonnegative bounded semicharacters of $S$ (nontrivial homomorphisms to the multiplicative semigroup $[0,1]$ ) that are $\mu$ - measurable for all $\mu \in \mathcal{M}(S)$ endowed with the topology of pointwise convergence.

Theorem 1. Let $S$ be an Abelian locally compact semitopological semigroup with unit $e$.

1) If $S$ has no nontrivial invertible elements and the space $S_{1}^{*}$ is path-connected then the Gelfand spectrum $\Delta \mathcal{M}(S)$ of the algebra $\mathcal{M}(S)$ is contractible.

2) Let $S$ has the form $P \cup\{e\}$ where $P$ is an open subsemigroup of a locally compact Abelian group $G$ and the unit $e$ of $G$ belongs to the closure of $P$. If $\Delta \mathcal{M}(S)$ is contractible, the space $S_{1}^{*}$ is path-connected.

Proof. 1) (cf. [1]). Denote by $1_{e}$ the characteristic function of the set $\{e\}$. Clearly, $1_{e} \in S_{1}^{*}$. Let $\left(\rho_{t}\right)_{t \in[0,1]}$ be a continuous path in $S_{1}^{*}$ such that $\rho_{0}=\mathbf{1}, \rho_{1}=1_{e}$ 
(1 denotes the unit semicharacter of $S$.) Define a continuous map $H: \Delta \mathcal{M}(S) \times$ $[0,1] \rightarrow \Delta \mathcal{M}(S)$ as follows:

$$
H(\varphi, t)(\mu)=\varphi\left(\rho_{t} \cdot \mu\right)
$$

where $\rho_{t} \cdot \mu$ means the product of a function and a measure in the sense of [3]. We show that $H$ is well-defined. Indeed, since $\rho_{t} \cdot(\mu * \nu)=\left(\rho_{t} \cdot \mu\right) *\left(\rho_{t} \cdot \nu\right)$ (see, e.g., [3, Chapter VIII, Section 3, Proposition 6]), we have $H(\varphi, t) \in \Delta \mathcal{M}(S)$. Moreover, by the inequality $\left(\varphi, \varphi_{0} \in \Delta \mathcal{M}(S), \mu \in \mathcal{M}(S)\right)$

$$
\left|H(\varphi, t)(\mu)-H\left(\varphi_{0}, t_{0}\right)(\mu)\right| \leq\left|\varphi\left(\rho_{t} \cdot \mu\right)-\varphi\left(\rho_{t_{0}} \cdot \mu\right)\right|+\left|\varphi\left(\rho_{t_{0}} \cdot \mu\right)-\varphi_{0}\left(\rho_{t_{0}} \cdot \mu\right)\right|
$$

the map $H$ is continuous because

$$
\left|\varphi\left(\rho_{t} \cdot \mu\right)-\varphi\left(\rho_{t_{0}} \cdot \mu\right)\right| \leq\|\varphi\|\left\|\rho_{t} \cdot \mu-\rho_{t_{0}} \cdot \mu\right\|=\left\|\left(\rho_{t}-\rho_{t_{0}}\right) \cdot \mu\right\| \leq \int_{S}\left|\rho_{t}-\rho_{t_{0}}\right| d|\mu| \rightarrow 0
$$

as $t \rightarrow t_{0}$ by the Lebesgue theorem.

Finally, if we define $\varphi_{1}(\mu):=\mu(\{e\})$, then $H(\varphi, 0)=\varphi$, and $H(\varphi, 1)=\varphi_{1}$, for all $\varphi \in \Delta \mathcal{M}(S)$. So, $\Delta \mathcal{M}(S)$ is contractible.

2) Since $\Delta \mathcal{M}(S)$ is contractible, there exists a continuous map $H: \Delta \mathcal{M}(S) \times$ $[0,1] \rightarrow \Delta \mathcal{M}(S)$ such that $H(\varphi, 0)=\varphi$, and $H(\varphi, 1)=\varphi_{1}$ for all $\varphi \in \Delta \mathcal{M}(S)$ where $\varphi_{1}(\mu):=\mu(\{e\})$. Let $\varphi_{0}(\mu):=\int_{S} d \mu\left(\varphi_{0} \in \Delta \mathcal{M}(S)\right)$. For $t \in[0,1]$ define $\rho_{t}: S \rightarrow \mathbb{R}_{+}$as follows:

$$
\rho_{t}(s)=\left|H\left(\varphi_{0}, t\right)\left(\delta_{s}\right)\right|
$$

where $\delta_{s}$ denotes the Dirac measure centered on $s \in S$. Then $\rho_{t}(e)=1$ and $\rho_{t}(s) \leq$ $\left\|H\left(\varphi_{0}, t\right)\right\|\left\|\delta_{s}\right\|=1$. Since $\delta_{a} * \delta_{b}=\delta_{a b}(a, b \in S)$, the map $\rho_{t}$ is a homomorphism from $S$ to $[0,1]$. By the result of Devinatz and Nussbaum [4] the restriction $\rho_{t} \mid P$ is continuous and we conclude that $\rho_{t} \in S_{1}^{*}$. Since the map $t \mapsto H\left(\varphi_{0}, t\right)$ is continuous, we get a continuous path $\left(\rho_{t}\right)_{t \in[0,1]}$ in $S_{1}^{*}$ such that $\rho_{0}=\mathbf{1}, \rho_{1}=1_{e}$. Therefore for every $\rho \in S_{1}^{*}$ we have a continuous path $\left(\rho \rho_{t}\right)_{t \in[0,1]}$ in $S_{1}^{*}$ from $\rho$ to $1_{e}$ which completes the proof.

Remark 1. The condition that $S$ has no nontrivial invertible elements is essential in theorem 1 as examples of locally compact Abelian groups show (see theorem 2 below). We conjecture that this condition is in fact necessary for $\Delta \mathcal{M}(S)$ to be contractible.

Remark 2 (cf. [1). The part 1 of theorem 1 remains valid (along with its proof) for every unital Banach subalgebra $\mathcal{R}$ of $\mathcal{M}(S)$ with the property: for some continuous path $\left(\rho_{t}\right)_{t \in[0,1]}$ in $S_{1}^{*}$ from 1 to $1_{e}$

$$
\forall \mu \in \mathcal{R}, \forall t \in[0,1] \quad \rho_{t} \cdot \mu \in \mathcal{R} .
$$

Examples. Let $S$ be an Abelian topological semigroup with invariant measure $m$ [5] and unit $e$ without nontrivial invertible elements (in particular, let $S$ be a Borel subsemigroup of a locally compact Abelian group with nonempty interior 
and without nontrivial subgroups, $e \in S$ ). Then the property (1) holds for the subalgebra $\mathcal{M}_{d}(S)$ of atomic measures on $S$, and for the subalgebra $L^{1}(S, m)+$ $\mathcal{M}_{d}(S)$ of all complex Borel measures that do not have a singular non-atomic part with respect to $m$; the property (1) holds also for the algebra $L^{1}(S, m)+\mathbb{C} \delta_{e}$. So the Gelfand spectra of these algebras are contractible. The same is true for images of aforementioned algebras with respect to the generalized Laplace transform in the sense of N. Bourbaki if the semigroup $\widehat{S}$ of all continuous bounded semicharacters of $S$ is complete (see [3, Chapter IX, subsection 5.7], and also [6], [7]).

Corollary 1 (cf. [1]). Let $S$ has no nontrivial invertible elements and the space $S_{1}^{*}$ is path-connected. Every unital Banach subalgebra $\mathcal{R}$ of $\mathcal{M}(S)$ with the property (1) is a Hermite ring in a sense that for every left invertible $n \times k$ matrix $\left(f_{i j}\right)$ with entries in $\mathcal{R}(k<n)$ there exists an invertible $n \times n$ matrix $\left(g_{i j}\right)$ with entries in $\mathcal{R}$ such that $g_{i j}=f_{i j}$ for all $1 \leq i \leq n$ and $1 \leq j \leq k$.

It follows from the remark 1 and [8, Theorem 3, p. 127].

Remark 3. For applications of results like corollary 1 to the problems in control theory see [1] and references there.

Corollary 2. Let $S$ be an Abelian locally compact semitopological semigroup with unit $e$ and without nontrivial invertible elements. If there is $\rho \in S_{1}^{*}$ such that $0<\rho(s)<1$ for all $s \in S \backslash\{e\}$ then for every unital Banach subalgebra $\mathcal{R}$ of $\mathcal{M}(S)$ with the property (1) the Gelfand spectrum $\Delta \mathcal{R}$ is contractible.

Indeed, in this case we have a continuous path $\left(\rho_{t}\right)_{t \in[0,1]}$ in $S_{1}^{*}$, where $\rho_{t}:=$ $\rho^{t /(1-t)}$ for $t \in[0,1), \rho_{1}:=1_{e}$, from 1 to $1_{e}$.

Theorem 2. For a nontrivial locally compact Abelian group $G$ the space $\Delta \mathcal{M}(G)$ is noncontractible.

Proof. Suppose that the Gelfand spectrum $\Delta=\Delta \mathcal{M}(G)$ is contractible. Then the one-dimensional Cech cohomology group $H^{1}(\Delta)$ with integral coefficients is trivial (see, e.g., 9, Chapter 2, section 2.1]). On the other hand, since $L^{1}\left(G_{d}\right)$ is a maximal group algebra in $\mathcal{M}(G)[10,8.2 .1]\left(G_{d}\right.$ denotes the group $G$ endowed with discrete topology), Theorem 8.1.3 from [10] (see also [2, Theorem 5.3.7]) implies that $H^{1}(\Delta)$ contains the isomorphic image of $H^{1}\left(\Delta L^{1}\left(G_{d}\right)\right)=H^{1}\left(\widehat{G_{d}}\right)$. If the dual group $\widehat{G_{d}}$ is connected, it is known $[10,8.3 .2]$ that $H^{1}\left(\widehat{G_{d}}\right)$ is isomorphic to $\widehat{G_{d}}=G$ and we have a contradiction with $H^{1}(\Delta)=0$. Now let $\widehat{G_{d}}$ be disconnected. Then the group $G_{d}$ contains a nontrivial finite subgroup $F$ (see, e.g., [11, (24.19)]). If $F \neq G$ the normalized Haar measure of $F$ is a nontrivial idempotent in $\mathcal{M}(G)$ and therefore $\Delta$ is disconnected by Shilov's Idempotent Theorem (and if $G=F$ this is obvious). This completes the proof.

We say that $S$ is an $M$-semigroup if every its semicharacter of the form $s \mapsto$ $\left|\varphi\left(\delta_{s}\right)\right|(\varphi \in \mathcal{M}(S))$ belongs to $S_{1}^{*}$. The proof of the part 2 of theorem 1 and results of [12, section 3] give us examples of $M$-semigroups. On the other hand, there are locally compact Abelian topological semigroups of idempotents which are not $M$-semigroups [12, subsection 2.4].

Theorem 3. Let $S$ be an Abelian locally compact semitopological $M$-semigroup with unit $e$ and without nontrivial invertible elements. Then $\operatorname{\Delta M}(S)$ is con- 
tractible if and only if the space $S_{1}^{*}$ is path-connected.

Indeed, the sufficiency follows from part 1 of theorem 1 . As regards the necessity, it can be proved in the same way as the part 2 of theorem 1 .

Acknowledgments. The paper was published in Semigroup Forum - 2019 - Vol. 98, No 1 - P. 209 -212; https://doi.org/10.1007/s00233-018-9974-x. The author is deeply indebted to the referee for very useful comments and suggestions that improve the paper.

\section{References}

[1] Sasane, A.: Extention to an invertible matrix in convolution algebras of measures supported in $[0 ;+\infty)$. In: Topics in Operator Theory: Advances and Applications. Birkhäuser, Basel. Vol. 202, 509 - 518 (2010)

[2] Graham, C. C. and McGehee, M. G.: Esseys in commutative harminic analysis. Springer-Verlag, New York, Heildelberg, Berlin (1979)

[3] Bourbaki, N.: Elements de mathematique. Livre VI. Integration. 2nd ed., Ch. 1 - 9. Hermann, Paris $(1965-1969)$

[4] Devinatz, A., Nussbaum A. E.: Real characters of certain semigroups with applications. Duke Math. J. 28 (2), 221 - 237 (1961)

[5] Mirotin, A. R.: Every Invariant Measure Semigroup Contains an Ideal which is Embeddable in a Group. Semigroup Forum. 59 (3), 354 - 361 ( 1999)

[6] Mirotin, A. R.: Positive semicharacters and the Laplace transform. Ukrainskii Matematicheskii Zhurnal, 44 (5), 647 - 653 (1992); Ukr Math J 44 (5), 582 - 588 (1992)

[7] Mirotin, A. R.: The Paley-Wiener theorem for cones in locally compact abelian groups. Izv. Vyssh. Uchebn. Zaved. Mat., no. 3, 35 - 44 ( 1995); Russian Math. (Iz. VUZ), 39:3, 33 - 42 (1995)

[8] Lin, V. Ya.: Holomorphic fiberings and multivalued functions of elements of a Banach algebra. Functional Analysis and its Applications. 7 (2), 122-128 (1973). English translation.

[9] Massey, W. S.: Homology and cohomology theory. Marcel Dekker Inc., New York and Basel (1978)

[10] Taylor, J. L.: Measure algebras. AMS, Providence, RI (1973)

[11] Hewitt, E., Ross, K.A.: Abstract Harmonic Analysis. Volume 1. SpringerVerlag, Berlin- Gettingen-Heidelberg (1963) 
[12] Baartz, A. P.: The measure algebra of a locally compact semigroup. Pacif. J. Math. 21 (2), 199 - 214 (1967) 